\title{
Sistematização da assistência de enfermagem (SAE) no período perioperatório: um relato de experiência
}

\author{
Systematization of nursing care in the perioperative period: an experience report
}

\section{Sistematización de la atención de enfermería en el período perioperatorio: un informe de experiencia}

Eduardo da Silva Gomes ${ }^{1 *}$, Evelyn Boeck dos Santos ${ }^{1}$, Fernanda Cristóvão Martins ${ }^{1}$, Angélica Dalmolin $^{1}$, Luiza Camila Jerke ${ }^{1}$, Adriana Franco Rechico ${ }^{2}$, Évilin Costa Gueterres ${ }^{1}$, Nara Marilene Oliveira Girardon-Perlini'.

\section{RESUMO}

Objetivo: Descrever a implementação da Sistematização da Assistência de Enfermagem (SAE) em uma unidade de cirurgia geral. Relato de experiência: Trata-se de experiência procedente da inserção de acadêmico de enfermagem em uma unidade de cirurgia geral durante estágio extracurricular em um hospital universitário de grande porte no interior do Rio Grande do Sul. O Processo de Enfermagem foi operacionalizado na prática clínica perioperatória pelo discente, que desenvolveu atividades assistenciais, administrativas e educativas junto a pacientes e familiares no pré e pós-operatório e aplicou instrumentos e protocolos que integram a SAE na instituição. Considerações finais: Com a experiência foi possível perceber a importância de implementar a SAE no desenvolvimento de atividades clínicas, gerenciais e educativas inerentes ao processo de cuidar em enfermagem no período perioperatório. A inserção do estudante no contexto da prática clínica representa uma oportunidade de aprofundamento e consolidação de conhecimentos teóricos, aprimoramento de habilidades técnico-assistenciais e desenvolvimento de autonomia para o exercício profissional.

Palavras-chave: Processo de enfermagem, Enfermagem perioperatória, Diagnóstico de enfermagem, Enfermagem médico-cirúrgica.

\section{ABSTRACT}

Objective: To describe the implementation of nursing care systematization in a general surgery unit. Experience report: This is an experience arising from the insertion of a nursing student in a general surgery unit during an extracurricular internship at a large university hospital in the interior of Rio Grande do Sul. The Nursing Process was operationalized in the clinical practice of perioperative nursing by the student, who developed assistance, administrative and educational activities with patients and family members in the pre and postoperative period and applied instruments and protocols that integrate the systematization in the institution. Final considerations: With the experience, it was possible to realize the importance of implementing the systematization in the development of clinical, managerial and educational activities inherent to the process of nursing care in the perioperative context. The insertion of the student in the context of clinical practice represents an opportunity to deepen and consolidate theoretical knowledge, improve technical-assistance skills and develop autonomy for professional practice.

Keywords: Nursing process, Perioperative nursing, Nursing diagnosis, Medical-surgical nursing.

\section{RESUMEN}

Objetivo: Describir la implementación de la sistematización de la atención de enfermería en una unidad de cirugía general. Informe de experiencia: Esta es una experiencia resultado de la inserción de un estudiante de enfermería en una unidad de cirugía general durante una pasantía extracurricular en un gran hospital universitario del interior de Rio Grande do Sul. El Proceso de Enfermería se operacionalizó en práctica clínica de enfermería perioperatoria por parte del estudiante, quien desarrolló actividades asistenciales, administrativas y educativas con pacientes y

1 Universidade Federal de Santa Maria (UFSM), Santa Maria - RS. *E-mail: eduardogomes703@gmail.com

2 Hospital Universitário de Santa Maria (HUSM), Santa Maria - RS. 
familiares en el período pre y posoperatorio y aplicó instrumentos y protocolos que integran la sistematización de la atención de enfermería en la institución. Consideraciones finales: Con la experiencia, fue posible darse cuenta de la importancia de implementar la sistematización de la atención de enfermería en el desarrollo de actividades clínicas, gerenciales y educativas inherentes al proceso del cuidado de enfermería en el contexto perioperatorio. La inserción del alumno en el contexto de la práctica clínica representa una oportunidad para profundizar y consolidar los conocimientos teóricos, mejorar las habilidades técnico-asistenciales y desarrollar la autonomía para la práctica profesional.

Palabras clave: Proceso de enfermería, Enfermería perioperatoria, Diagnóstico de enfermería, Enfermería médicoquirúrgica.

\section{INTRODUÇÃO}

A Sistematização da Assistência de Enfermagem (SAE) é uma metodologia científica que estrutura os serviços de enfermagem no que tange a organização do trabalho, recursos humanos e instrumentos assistenciais. Conforme a Resolução no 358/2009 do Conselho Federal de Enfermagem (COFEN), a SAE deve ser implementada em todos os ambientes em que ocorram o cuidado de enfermagem, por meio de um suporte teórico que possibilite a operacionalização do Processo de Enfermagem (PE) (TANNURE MC e GONÇALVES AMP, 2011; CONSELHO FEDERAL DE ENFERMAGEM, 2009).

O cuidado de enfermagem sistematizado oportuniza uma melhora na qualidade da assistência e maior segurança aos pacientes, ao possibilitar a execução de um cuidado holístico, integral e contínuo, com base em evidências científicas. Na perspectiva dos profissionais, a SAE permite a aplicação de conhecimentos técnico-científicos, nas dimensões assistenciais, educacionais e gerenciais, proporcionando maior autonomia e satisfação profissional (TANNURE MC e GONÇALVES AMP, 2011).

No contexto das unidades perioperatórias, a assistência de enfermagem possui a finalidade de promover a recuperação do paciente submetido a um procedimento cirúrgico. As ações de enfermagem devem transcorrer do pré ao pós-operatório, contemplando orientações aos pacientes e sua família, a fim de preparálos para o procedimento cirúrgico e regresso ao domicílio, o que caracteriza o cuidado perioperatório como um processo dinâmico, complexo e interativo (SOUZA LR, et al., 2010; SANTOS IMBE, et al., 2020).

Em vista disso, a sistematização do cuidado nesses ambientes é essencial, pois possibilita o planejamento da assistência de enfermagem desde a admissão do paciente, até a alta hospitalar (SANTO IMBE et al, 2020). Para isso, o raciocínio clínico dos profissionais deve estar amparado em referenciais teóricos específicos da enfermagem, com vistas a implementar intervenções coerentes e acuradas (TANNURE MC e GONÇALVES AMP, 2011; CONSELHO FEDERAL DE ENFERMAGEM, 2009).

No entanto, a enfermagem ainda possui dificuldades em implementar a SAE no seu cotidiano de trabalho, em razão da falta de incentivo e interesse institucional, da sobrecarga laboral, do desconhecimento acerca da importância da operacionalização do PE, bem como da falta de domínio no que se refere ao suporte teórico que subsidia o cuidado sistematizado (REIS GS, et al., 2016; ADAMY EK e TOSATTI M, 2012).

Dentre os fatores que contribuem e limitam a efetivação da SAE em unidades cirúrgicas, sinaliza-se a incipiência da produção científica. Em estudo que objetivou identificar e analisar artigos científicos que descreveram a SAE em uma das fases do período perioperatório, foi evidenciada uma lacuna no conhecimento acerca dos modelos de operacionalização da SAE na atenção ao paciente cirúrgico (PREARO M e FONTES CMB, 2019).

Logo, percebe-se a necessidade de desenvolver estudos sobre essa metodologia de trabalho, com a finalidade de fortalecer e caracterizar o corpo de saberes específicos da enfermagem, colaborando para sua consolidação enquanto ciência e disciplina (SOUZA GB, et al., 2020).

Ressalta-se também, a imprescindibilidade de ampliar os conhecimentos relativos a SAE e ao PE durante a formação profissional do enfermeiro, com o intuito de proporcionar subsídios para uma atuação efetiva (CAVEIÃO C, et al., 2020). 
Nessa perspectiva, o Programa de Formação Complementar em Enfermagem (PROFCEN), da Universidade Federal de Santa Maria (UFSM), permite ao estudante sua inserção nos cenários práticos da assistência em saúde, favorecendo o desenvolvimento de habilidades técnico-científicas, bem como aprofundamento teórico dos conhecimentos (UFSM, 2015). Assim, constitui-se como uma possibilidade de ampliar saberes acerca da implementação da SAE na prática laboral da profissão.

A partir do exposto, o presente estudo tem como objetivo descrever a implementação da SAE em uma unidade de cirurgia geral.

\section{RELATO DE EXPERIÊNCIA}

Trata-se de um estudo qualitativo, na modalidade relato de experiência, procedente da inserção de acadêmico de enfermagem no programa de ensino-extensão PROFCEN, nos meses de janeiro e fevereiro de 2020, totalizando 170 horas de atividades extracurriculares. A atuação do discente teve supervisão direta de uma enfermeira responsável e indireta de um docente do Departamento de Enfermagem da UFSM.

O local da experiência foi a Unidade de Cirurgia Geral (UCG) de um hospital universitário de grande porte do Rio Grande do Sul. A UCG possui 52 leitos de internação e presta cuidados no pré e pós-operatório a pacientes em procedimentos cirúrgicos de baixa, média e alta complexidade em diversas especialidades. $\mathrm{Na}$ unidade, a equipe de enfermagem é composta por 54 profissionais, sendo 17 enfermeiros e 37 técnicos de enfermagem.

Como estratégia de inserção na UCG, em um primeiro momento, realizou-se a aproximação com a unidade e os profissionais atuantes no setor. Com isso, foi possível conhecer a rotina de trabalho da equipe de enfermagem, bem como a estrutura física e organizacional da instituição. Nos dias subsequentes, o graduando desenvolveu atividades assistenciais, administrativas e educativas inerentes a prática laboral da enfermagem no contexto dos cuidados perioperatórios.

A instituição utiliza como referencial teórico para a SAE a Teoria de Necessidades Humanas Básicas de Wanda Horta. Todas as etapas do PE encontram-se implementadas e informatizadas, possuindo como suporte a taxonomia NANDA International (NANDA-I) e a Nursing Interventions Classification (NIC), para as etapas de diagnóstico e intervenção, respectivamente. As evoluções e anotações de enfermagem são registradas em sistema eletrônico.

A primeira atividade no turno de trabalho consistia em participar da passagem de plantão. Para esse momento, os profissionais e estudantes se reuniam em um local do posto de enfermagem destinado para essa prática. A atividade era guiada por um instrumento padronizado e impresso, o qual continha informações sobre os dados de identificação dos pacientes, condições clínicas e procedimentos cirúrgicos realizados ou agendados, bem como cuidados de enfermagem específicos para cada paciente.

Após a passagem do plantão, era feita a visita diária ao leito dos pacientes em pré e pós-operatório. Essa prática era composta pelas etapas de avaliação e coleta de dados do PE, composta pela anamnese e exame físico, com vistas a obter informações precisas acerca do quadro de saúde dos pacientes e direcionar a atuação dos profissionais de enfermagem com base no julgamento clínico.

Com os dados clínicos reunidos, eram identificados os diagnósticos de enfermagem e definia-se as intervenções prioritárias para cada paciente. Esse processo sucedia-se por meio do sistema informatizado da instituição, o qual permite realizar as ligações entre as taxonomias NANDA-I e NIC. As ligações eram realizadas de acordo com as necessidades apresentadas pelos pacientes nas dimensões psicobiológicas, psicossociais e/ou psicoespirituais. Ressalta-se que os diagnósticos de enfermagem, assim como as prescrições, eram impressos e disponibilizados também no prontuário físico dos pacientes.

Para além da efetivação das etapas do PE, os pacientes eram avaliados diariamente quanto ao risco de desenvolver lesão por pressão, o grau de dependência dos cuidados da equipe de enfermagem e uso de recursos e tecnologias, como cateteres e drenos. Para essas finalidades, aplicava-se a escala Braden e 0 Sistema de Classificação de Pacientes, respectivamente. Esse último, era aplicado com o intuito de subsidiar o cálculo de dimensionamento de recursos humanos na UCG, tendo em vista o grande número de leitos disponíveis no setor e o restrito número de profissionais de enfermagem atuantes no local. 
No que concerne aos procedimentos técnicos desenvolvidos pelo acadêmico, estes eram caracterizados na maioria das vezes, como cuidados complexos e privativos do enfermeiro, dentre eles a passagem de cateteres nasoenterais e vesicais de demora, aspiração traqueal e manejo clínico de lesões de difícil cicatrização com uso de coberturas especiais. Todos esses procedimentos são padronizados por meio de Procedimentos Operacionais Padrão (POP) que integram a SAE.

Era efetuada também, a admissão de novos pacientes na unidade, provenientes do domicílio ou de outros setores do hospital. Nesse último contexto, os dados referentes ao quadro clínico dos pacientes eram facilmente acessados no sistema eletrônico.

Assim, os diagnósticos e intervenções de enfermagem planejadas em outros setores da instituição eram passíveis de visualização pela equipe da UCG, o que possibilitava a continuidade da assistência. Já na admissão de pacientes provenientes do domicílio, era executada a coleta de dados de forma integral, e posterior operacionalização das demais etapas do PE, quais sejam: diagnóstico, planejamento, implementação e avaliação.

Com relação aos pacientes que iriam ser submetidos a intervenção cirúrgica, a instituição possui como rotina a aplicação de um Checklist, com vistas a garantir segurança ao paciente no transcorrer do período perioperatório. Ao encaminhar os pacientes para o bloco cirúrgico, esse instrumento era preenchido parcialmente com dados de identificação, procedimento cirúrgico a ser realizado e confirmação de jejum préoperatório. Com a chegada ao paciente no centro cirúrgico, o registro do Checklist é finalizado pela equipe multiprofissional na presença do paciente.

Destaca-se também, as intervenções educativas desenvolvidas junto aos pacientes e seus familiares que possuíam a finalidade de orientá-los e instruí-los ao longo da hospitalização. Essas intervenções, eram realizadas em conjunto com as etapas de avaliação e/ou coleta de dados do PE, ou em casos de dúvidas e questionamentos por parte dos pacientes e/ou familiares. As ações educativas contemplavam informações sobre os procedimentos realizados, a cirurgia e dispositivos em uso pelos pacientes.

Nos momentos finais dos turnos de trabalho eram realizadas as evoluções de enfermagem. Assim, registrava-se as respostas dos pacientes e os resultados alcançados frente as intervenções implementadas, o que possibilitava o planejamento dos cuidados de enfermagem nos turnos subsequentes.

Para a estruturação das evoluções, tem-se como norma o uso do acrônimo SOAP, que descreve o registro clínico a partir do Subjetivo, Objetivo, Avaliação e Plano terapêutico. Por fim, realizava-se a troca de plantão com os profissionais de enfermagem do turno seguinte, repassando as informações necessárias para a continuidade da assistência aos pacientes.

\section{DISCUSSÃO}

A implementação da SAE no período perioperatório permitiu a inter-relação da assistência entre os períodos pré, trans e pós-operatório, contemplando toda a experiência cirúrgica com pacientes e familiares (FENGLER FC e MEDEIROS CRG, 2020). Nesse sentido, configurou-se como uma metodologia capaz de nortear a atuação da equipe de enfermagem, por meio de instrumentos e protocolos, além de contribuir para um cuidado individualizado e efetivo.

Dentre os motivos pelo qual isso foi possível, destaca-se a adoção de um referencial teórico coerente, que permitiu a aplicação dos conhecimentos técnico-científicos na prática laboral da equipe de enfermagem (TANNURE MC e GONÇALVES AMP, 2011; CONSELHO FEDERAL DE ENFERMAGEM, 2009).

A Teoria de Necessidades Humanas Básicas pressupõe que a enfermagem ao assistir o indivíduo deve considerá-lo como um ser único, o qual necessita ser cuidado de forma integral, respeitando sua individualidade e seu contexto sociocultural. Em situações de desequilíbrio da saúde, o paciente e sua família possuem necessidades básicas que precisam ser atendidas para alcançar o completo bem-estar. Para isso, a enfermagem deve implementar ações assistenciais, gerenciais e educativas (HORTA WA, 1974). 
Segundo Horta WA (1974), a assistência de enfermagem precisa ser norteada pelo PE, o qual é composto por etapas inter-relacionadas e recorrentes. Com vistas a facilitar a implementação dessas etapas na prática clínica, os sistemas classificatórios de enfermagem podem ser empregados, com destaque para as taxonomias NANDA-I, NIC e Nursing Outcomes Classification (NOC).

A NANDA-I proporciona a descrição das respostas humanas frente a uma situação de adoecimento com base no julgamento clínico dos enfermeiros (HERDMAN TH e KAMITSURU S, 2018). A NIC, possui intervenções de enfermagem possíveis de serem implementadas para alcançar resultados positivos e/ou solucionar problemas de saúde de um indivíduo, família ou comunidade (BULECHEK GM, et al., 2016).

Esses sistemas funcionam de forma complementar a NOC, que objetiva avaliar a efetividade das intervenções ao longo de um continuum (MOORHEAD S, et al., 2008). No entanto, seu uso ainda não é realidade na instituição do estudo, o que pode constituir-se em um ponto fragilizador no que se refere à avaliação e documentação da efetividade das intervenções de enfermagem.

Outro aspecto de destaque está na utilização de um instrumento impresso para a passagem de plantão. Conforme evidenciado na literatura, protocolos e ferramentas padronizadas para a passagem de turno possibilitam a troca de informações entre os profissionais de enfermagem de forma eficiente, garantindo a continuidade da assistência e configurando-se como recursos promotores de um cuidado seguro (CORPOLATO RC, et al., 2019).

Nessa mesma lógica, o emprego do Checklist cirúrgico e das escalas preditivas de risco constituem-se também como promotores de segurança aos pacientes e trabalhadores. Na "Aliança Mundial para a Segurança do Paciente", promovida pela Organização Mundial da Saúde (OMS), uma das recomendações é referente ao uso de ferramentas sistematizadas, antes, durante e após a indução anestésica (ORGANIZAÇÃO MUNDIAL DA SAÚDE, 2009).

Ademais, em um estudo que objetivou conhecer a percepção dos profissionais sobre o uso do Checklist cirúrgico, foi evidenciado que a enfermagem percebe o recurso como potencializador da comunicação entre os membros da equipe, além de reduzir a chance de eventos adversos (GOMES CDPP, et al., 2016).

Percebe-se que a SAE envolve não somente a operacionalização do PE, mas também a organização e estruturação do trabalho da enfermagem quanto aos recursos humanos e materiais, instrumentos e métodos laborais. Assim, a formulação e utilização de protocolos e POPs durante as atividades assistenciais também se constituem como formas de sistematizar o cuidado e favorecer uma prática segura para pacientes e profissionais.

Outra atividade que reflete os benefícios de um cuidado sistematizado são as intervenções educativas. Nesse sentido, o PE ao requerer que o profissional realize coleta de dados e avaliação clínica dos pacientes de forma contínua, possibilita a formação do vínculo entre equipe, paciente e família, facilitando a implementação das orientações. Ressalta-se que a educação em saúde era um dos itens que constava na prescrição de enfermagem, o que pode ser um fator favorável à sua realização pelos membros da equipe.

Segundo Paiva (2017), as orientações educativas contribuem para a redução da ansiedade e complicações no transcorrer do período perioperatório, configurando-se como um importante recurso da equipe de enfermagem. Além disso, são promotoras da independência e da autonomia para o cuidado e o autocuidado (DALMOLIN A, et al., 2016).

Dentre as limitações da experiência, destaca-se a carência de profissionais de enfermagem na UCG, pois foi possível observar a sobrecarga de trabalho dos profissionais, devido a demanda de tarefas e cuidados a serem desempenhados, o que pode ser um aspecto a contribuir para um cuidado menos qualificado em alguns momentos. Ainda, percebeu-se a necessidade de realização de funções que não são inerentes ao núcleo de enfermagem, as quais envolvem demandas na área gerencial que poderiam ser desenvolvidas por outras categorias profissionais. Tais aspectos podem reverberar em fragilidades no que tange ao tempo disponibilizado a implementação da SAE, uma vez que esta podem ser consideradas de menor importância (SOUSA BVN, et al., 2020). 
Com a experiência foi possível perceber a importância de implementar a SAE no desenvolvimento de atividades clínicas, gerenciais e educativas inerentes ao processo de cuidar em enfermagem no contexto perioperatório, haja vista que contribui para uma assistência individualizada e efetiva, com base em evidências clínicas. Destaca-se que os protocolos de cuidados se configuraram como instrumentos promotores de uma assistência de enfermagem mais segura e organizada.

No entanto, revela-se que a sobrecarga de atividades no serviço constitui-se em um ponto fragilizador no que se refere a implementação da SAE na prática laboral da enfermagem. Por fim, ressalta-se que a inserção do estudante no contexto da prática clínica representa uma oportunidade de aprofundamento e consolidação de conhecimentos teóricos, aprimoramento de habilidades técnico-assistenciais e promoção de autonomia para o exercício profissional.

\section{REFERÊNCIAS}

1. ADAMY EK, TOSATTI M. Sistematização da assistência de enfermagem no período perioperatório: visão da equipe de enfermagem. Revista de Enfermagem UFSM, 2012; 2(2):300-310.

2. BULECHEK GM, et al. Classificação das Intervenções de Enfermagem (NIC). 6 ed. Rio de Janeiro: Elsevier, 2016.

3. CAVEIÃO C, et al. Sistematização da assistência e processo de enfermagem: conhecimento de estudantes de enfermagem. Revista de Pesquisa: Cuidado é Fundamental, 2020; 12(1): 1093-1098

4. CONSELHO FEDERAL DE ENFERMAGEM. Resolução Conselho Federal de Enfermagem (COFEN) 358/2009. Brasília: COFEN, 2009.

5. CORPOLATO RC, et al. Padronização da passagem de plantão em Unidade de Terapia Intensiva Geral Adulto. Revista Brasileira de Enfermagem, 2019; 72(Suppl 1): 95-102.

6. DALMOLIN A, et al. Vídeo educativo com recurso para educação em saúde a pessoas com colostomia e familiares. Revista Gaúcha de Enfermagem Online, 2016; 37(spe): e68373.

7. FENGLER FC, MEDEIROS CRG. Sistematização da assistência de enfermagem no período perioperatório: análise de registros. Revista SOBECC, 2020; 25(1).

8. GOMES CDPP, et al. Percepção de uma equipe de enfermagem sobre a utilização do checklist cirúrgico. Revista SOBECC, 2016; 21(3).

9. HERDMAN TH, KAMITSURU S. Diagnósticos de enfermagem da NANDA: definições e classificação 2018-2020. 11 ed. Porto Alegre: Artmed, 2018.

10. HORTA WA. Enfermagem: teoria, conceitos, princípios e processo. Revista da Escola de Enfermagem da Universidade de São Paulo, 1974; 5(1) 7-15.

11. MOORHEAD S, et al. Classificação dos Resultados de Enfermagem NOC. 3 ed. Porto Alegre: Artmed, 2008.

12. ORGANIZAÇÃO MUNDIAL DA SAÚDE. Aliança Mundial para a Segurança do Paciente. Segundo desafio global para a segurança do paciente: cirurgias seguras salvam vidas. Rio de Janeiro: Organização Mundial da Saúde, 2009.

13. PAIVA BC, et al. Avaliação da efetividade da intervenção com material educativo em pacientes cirúrgicos: revisão integrativa da literatura. Revista SOBECC, 2017; 22(4).

14. PREARO M, FONTES CMB. Sistematização da assistência de enfermagem na sala de recuperação pós anestésica: revisão integrativa. Enfermagem em Foco, 2019; 135-140.

15. REIS GS, et al. Sistematização da assistência de enfermagem: vantagens e dificuldades na implantação. Arquivos médicos dos Hospitais e Faculdade de Ciências Médicas Santa Casa São Paulo, 2016; 61:128-32.

16. SANTOS IMBE, et al. Sistematização da Assistência de Enfermagem Perioperatória (SAEP): Reflexos da aplicabilidade no processo de cuidar. Revista Eletrônica Acervo Saúde, 2020; (43): e2945.

17. SOUSA BVN, et al. Benefícios e limitações da sistematização da assistência de enfermagem na gestão em saúde. Journal of Nursing and Health, 2020; 10(2): e20102001.

18. SOUZA GB, et al. Sistematização da assistência de enfermagem e processo de enfermagem: conhecimento de graduandos. Brazilian Journal of Health Review, 2020; 3(1): 1250-1271.

19. SOUZA LR, et al. Os benefícios da visita pré-operatória de enfermagem para o cliente cirúrgico: revisão sistemática da literatura. Revista de Pesquisa Cuidado é Fundamental Online, 2010; 2(2): 797-806.

20. TANNURE MC, GONÇALVES AMP. Sistematização da assistência de enfermagem: guia Prático. 2nd ed. Rio de Janeiro: Guanabara Koogan, 2011.

21. UNIVERSIDADE FEDERAL DE SANTA MARIA. 2015. In: Programa de Formação Complementar em Enfermagem PROFCEN. Disponível em: https://portal.ufsm.br/projetos/publico/projetos/view.html?idProjeto=48361 Acesso em: 15 out. 2020. 